\title{
Deux carnets et une orthographe : genèse, publication et lecture de William Shakespeare
}

\author{
Guy Rosa
}

\section{(2) OpenEdition}

10 Journals

Édition électronique

URL : http://journals.openedition.org/genesis/2897

DOI : 10.4000/genesis.2897

ISSN : 2268-1590

Éditeur :

Presses universitaires de Paris Sorbonne (PUPS), Société internationale de génétique artistique littéraire et scientifique (SIGALES)

Édition imprimée

Date de publication : 5 décembre 2017

Pagination : $71-81$

ISBN : 979-10-231-0580-3

ISSN : $1167-5101$

\section{Référence électronique}

Guy Rosa, "Deux carnets et une orthographe : genèse, publication et lecture de William Shakespeare », Genesis [En ligne], 45 | 2017, mis en ligne le 15 décembre 2018, consulté le 12 janvier 2021. URL : http://journals.openedition.org/genesis/2897 ; DOI : https://doi.org/10.4000/genesis.2897 


\section{Deux carnets et une orthographe : genèse, publication et lecture de William Shakespeare}

Guy Rosa

$\mathrm{E}$ n 1937, procuré par Cécile Daubray, le trente-septième volume de la collection des EEuvres complètes de Hugo, lancée en 1902 et confiée aux presses de l'Imprimerie nationale - de là qu'on l'appelle l'IN -, inaugure une nouvelle lecture de William Shakespeare, pour autant qu'il en ait eu une auparavant et qu'il s'agisse encore du livre publié en 1864. Cécile Daubray n'innove ni par l'amélioration du texte, assez mal établi, ni par le commentaire ou l'annotation - il n'y en a pas -, mais par l'ajout de textes, inédits pour la plupart, qui occupent plus de la moitié du volume. Ce sont, sans surprise, une demi-douzaine de «chutes» du travail de rédaction, des notes documentaires d'une destination plus ou moins problématique et les éléments d'un dossier formé en vue d'un autre projet dont Hugo a distrait une partie pour William Shakespeare, mais l'essentiel, en volume et en importance, vient d'ailleurs.

En 1860, Yves Gohin l'a montré1, la longue méditation que demande la reprise des Misérables génère un mouvement d'écriture en prose abstraite, philosophique et religieuse, mouvement puissant au point d'interrompre le travail des Misérables et neuf pour Hugo qui avait jusque-là confié ces matières à la poésie lyrique - Les Contemplations -, ou épique - La Fin de Satan, L’Âne, La Révolution. Il court jusqu'en 1865, sous-jacent aux œuvres publiées : Les Misérables, où il affleure dans les grandes digressions; William Shakespeare, qui en relève tout entier - mais pour s'en extraire ou pour s'y absorber, c'est toute la question -; Les Travailleurs de la mer, d'abord intitulé L'Abîme. Mais ce courant d'écriture vient au jour en tant que tel et directement dans une quinzaine de textes inachevés, longs fragments ou œuvres avortées, tous inédits à la mort de Hugo. Le premier en date et le plus important, intitulé Philosophie, était destiné à servir de préface aux Misérables ou à l'ensemble de son œuvre, ou encore à figurer dans des Mémoires de ma vie intellectuelle. L'IN le place dans le Reliquat des Misérables, sous le titre Préface philosophique, avec le texte intitulé
Les Fleurs, rebaptisé L'Âme, que Hugo avait retiré du roman et réservé «pour mon travail sur l'âme». Le dernier, $\mathrm{La}$ Mer et le vent, est inséré dans Les Travailleurs de la mer, «sans bonnes raisons» dit Yves Gohin. L'IN annexe le reste à William Shakespeare.

Meurice et Vacquerie n'y avaient pas touché dans l'édition ne varietur de 1882, se contentant d'y joindre la «Préface pour la traduction de Shakespeare de FrançoisVictor Hugo » jusqu'alors publiée avec cette traduction ${ }^{2}$. Ils avaient cependant confectionné, dans la série des œuvres posthumes, un volume Post-scriptum de ma vie, où un florilège de brèves réflexions de «philosophie» et de critique littéraire, extraites des dossiers de Hugo sans aucun souci de leurs dates, entrelardait d'une garniture quatre de ces longs inédits, Utilité du Beau, Le Goût, Du génie et Promontorium somnii. On trichait bien un peu en affirmant que «ces manuscrits de prose se composent d'assez forts cahiers», mais Post-scriptum de ma vie n'était rattaché d'aucune manière à William Shakespeare. Cécile Daubray s'affranchit de ce scrupule.

Le volume de l'IN intitulé William Shakespeare contient, à la suite de l'œuvre et de la préface pour la traduction de François-Victor Hugo, un «Reliquat de William Shakespeare» (155 pages) et des «Notes de travail» (31 pages). Il ne s'arrête pas là, il continue, ou recommence, par Post-scriptum de ma vie (154 pages). C'est le même livre, est-il annoncé, que celui de l'édition ne varietur, amputé de «certains récits [Promontorium somnii et À Reims] que nous avons restitués au Reliquat de William Shakespeare [...] remplacés ici par

1. Dans son édition des «Proses philosophiques des années 1860-1865» (V. Hugo, Euvres complètes, éd. J. Seebacher et G. Rosa, vol. Critique, Paris, Laffont, coll. «Bouquins », 1985, rééd. 2002 [désormais Laffont]), mais aussi dès son étude Sur l'emploi des mots «immanent» et «immanence $»$ chez Victor Hugo, Archives des lettres modernes, coll. «Archives hugoliennes », $\mathrm{n}^{\circ}$ 6, Minard, 1968.

2. Voir ci-dessous la note 26. 
des chapitres inédits [L'Infiniment petit et La civilisation $]^{3}$ ». Au total, un ensemble considérable de textes, amalgamés à William Shakespeare et finissant par le déborder - 340 pages contre les 230 de l'œuvre -, lui était ainsi indûment assimilé. Voire frauduleusement car, joignant la parole au geste, la présentation du Reliquat en justifie ainsi la présence :

Un carnet de Victor Hugo porte, à la date du 21 mai 1864, cette indication :

«J'ai fait le rangement des papiers et le ménage des copeaux qui me restent de mon livre William Shakespeare.»

Les papiers, forts cahiers détachés par Hugo du manuscrit même, constituent aujourd'hui ce Reliquat : À Reims, - Les Génies appartenant au peuple, - Sur Homère, - Beethoven, - Le Goût, - Promontorium Somnii, - Le Tyran, - La Bible. L'Angleterre, - Les Traducteurs.

Et Cécile Daubray conclut :

[...] nous avons restitué à William Shakespeare ce qui lui appartenait et rétabli le texte intégral ${ }^{4}$.

Rien n'est exact. Depuis les vers d'écolier, pas de cahiers chez Hugo dont le mépris pour les auteurs à cahiers s'exprime précisément dans William Shakespeare; à plus forte raison n'y a-t-il aucune trace que ces cahiers ou d'autres aient jamais été attachés au manuscrit de William Shakespeare, «détachés » moins encore. L'inventaire après décès ne mentionne aucun «reliquat» de William Shakespeare, ni non plus d'ailleurs d'aucune œuvre à l'exception des Misérables; il ne laisse pas davantage soupçonner qu'aucun dossier ait contenu des «papiers» se rapportant à William Shakespeare. Si Hugo a classé ceux que l'IN publie, impossible de dire où ni comment. Il fallait plus que de la hardiesse, de l'effronterie, pour insinuer qu'on rétablissait un William Shakespeare complet et intégral en mettant à sa suite tout ce fatras de textes très inégalement liés à lui et que Hugo avait abandonnés sans jamais songer à les publier avec ce livre.

Ce que les proses philosophiques des années 1860-1865 perdent à leur dispersion aux trois reliquats des œuvres de même date, on le mesure en les lisant sous la conduite d'Yves Gohin dans le volume de l'édition Bouquins où il les a réunies. Mais le dommage est moindre que celui infligé par leur greffe sur le corps de William Shakespeare.
Cette greffe suggère d'abord une composition du livre hasardeuse, peut-être précipitée, en tout cas obscure. S'il faut lui restituer des «chapitres », c'est qu'ils lui manquent et n'y ont pas été intégrés, ou en ont été retirés, sinon à tort du moins pour des raisons peut-être fragiles. Elle aggrave aussi le disparate apparent de l'œuvre par l'hétéroclite de nouveaux objets : les visions du songe et du rêve, le tyran, la Bible, les traducteurs, le voyage du sacre à Reims en 1825 , etc. À force d'ajouter des fragments au livre, le livre luimême s'éparpille en fragments. Surtout William Shakespeare était de la sorte exposé à un mode de lecture nouveau : non plus celui d'une œuvre littéraire, totalité fermée exerçant des effets intellectuels, émotifs et spirituels concertés, mais celui que demande une collection de propositions abstraites et traductibles valant chacune par elle-même. C'est ce que dit le mot de somme, désormais appliqué à William Shakespeare par tous les commentateurs. Somme qu'on dit testamentaire, religieuse ou philosophique, «somme du romantisme ${ }^{5}$ », corrige Pierre Albouy à juste titre, mais somme toujours. De la littérature, dessaisie, William Shakespeare passait sous la juridiction de l'histoire des idées.

Longtemps l'IN bénéficia d'une autorité incontestée6. Son William Shakespeare à rallonges s'imposa. Toutes les éditions, à l'exception de «Bouquins », le reproduisent; tous les commentaires, renonçant à montrer la perspective générale du texte et à comprendre son propos, s'appliquent à élucider telle ou telle des idées de Hugo «au temps de William Shakespeare» et à les confronter à celles d'autres penseurs ou de Hugo en d'autres temps. Les meilleurs furent victimes de cette vulgate. Albouy lui-même conclut sa présentation du livre ${ }^{7}$ par une belle page sur sa puissance poétique mais, pour le reste, en analyse les contenus, avec

3. William Shakespeare; Euvres complètes de Victor Hugo, Philosophie - II, Albin Michel, Imprimerie nationale, librairie Ollendorff, 1937 [désormais IN], p. 467.

4. Ibid., p. 249.

5. Pierre Albouy, La Création mythologique chez Victor Hugo, Paris, José Corti, 1968 , p. 51 et 71 .

6. Jusqu'à ce que les travaux de R. Journet et G. Robert et la découverte par J. Seebacher de l'inventaire après décès des manuscrits finissent par discréditer les reliquats de l'IN. Voir ci-dessous l'entretien «Hugo de l'écrit au livre (suite)».

7. Victor Hugo, Euvres complètes, édition chronologique sous la direction de Jean Massin, Club Français du Livre [désormais CFL], t. XII, 1969, p. 127-148. 
d'ailleurs une intrépidité intellectuelle toute hugolienne ; il laisse cependant ouvertement de côté la Révolution, le Progrès, le lien entre les esprits et les masses, le devoir fait aux poètes de se mettre au service du vrai ${ }^{8}$. C'est explicable : tout cela, isolé du reste et devenu «idéologie», semble connu, et plutôt trop que pas assez. C'est pourtant regrettable : il n'y a pas, dans l'œuvre entière de Hugo, d'hymne plus ardent à la Révolution, 93 et Terreur compris, d'affirmation plus forte que romantisme et socialisme ne font qu'un, d'injonction plus subversive faite aux historiens de renvoyer aux oubliettes de l'histoire les rois, les reines, tous les puissants, et de promouvoir ceux dont l'effort met l'humanité à hauteur d'elle-même : écrivains et philosophes, savants et inventeurs, réformateurs et bienfaiteurs, tous les croyants. C'est aussi scientifiquement aventureux.

Jacques Seebacher le fait voir ${ }^{9}$, dont 1'intelligence conduit à leurs dernières conséquences les leçons de l'IN. Étudiant l'un des textes accolés à William Shakespeare, Utilité $d u$ Beau10, et y trouvant mieux assurée la continuité de la pensée de Hugo en matière d'esthétique - l'identité du fond et de la forme -, il commence par affaiblir la pertinence de la composition du livre :

En règle générale, Hugo compose par panneaux séparés dont l'assemblage définitif ne se fait que pour la publication, à l'image des tacticiens qui réservent toujours la plus grande souplesse entre ce qu'ils appellent leur «intention» et ce qu'ils nomment «la répartition des moyens et l'articulation des missions $\gg 11$.

Observons que cette «règle générale», déduite des exceptions qui la respectent 12 , autorise à mettre sur le même plan l'œuvre publiée et le fragment inédit, et rhabillons la métaphore en civil : il peut y avoir du jeu, un écart, une béance pourquoi pas, entre les idées de l'auteur et leur expression soumise aux besoins de la cause comme l'est William Shakespeare, sinon livre de circonstance du moins dépendant d'elles du simple fait qu'il est publié. Hugo met donc en réserve des textes tels qu'Utilité $d u$ Beau et c'est ce qui les rend précieux : «nous pouvons utiliser ces pages comme les témoins les plus intimes du génie hugolien. » En l'espèce, Utilité du Beau qui pose la bienfaisance du Beau quelle que soit l'intention de l'artiste, fût-elle condamnable, présentait une «contrepartie» aux injonctions militantes de William Shakespeare, pour ne pas dire une contradiction avec elles, qui explique sa mise à l'écart, et le fait préférer :

Ce génie double maîtrise toujours ses antithèses, mais n'en fait souvent paraître qu'un des termes, selon l'opportunité politique, se réservant de produire le reste au moment où, l'histoire ayant changé de cours, le génie devra jeter d'autres forces dans la bataille. C'est pourquoi des textes de cette nature sont peut-être plus importants pour la connaissance de Hugo que ceux qu'il a publiés $[\ldots]^{13}$.

C'était aller trop loin. À ce compte, plus importants encore pourraient être ceux qu'il n'a pas écrits. Le sentant, Jacques Seebacher, avant de conclure, se rétracte : «Peu nous importe maintenant que Hugo n'ait pas publié ce texte avec William Shakespeare $[\ldots]^{14}$.» Et voici jeté après usage l'argument initial tiré de la genèse du livre. Jacques Seebacher fait bien de s'en débarrasser : il était fragile, plus encore qu'il ne croyait.

Car l'opération éditoriale de l'IN étayait une logique solide sur des fondations bâclées. Elle impliquait une certaine représentation de la formation matérielle du livre : l'existence en marge de William Shakespeare de ces cahiers détachés du manuscrit devait résulter d'un choix hâtif qui expliquait aussi l'hétérogénéité des développements finalement retenus. Le même geste prononçait tout à la fois et confortait l'une par l'autre la transitivité discursive du texte, l'illisibilité de sa composition et la nature de sa

8. «D'actualité, lors de la Libération et des proclamations sur la littérature engagée, à nouveau à l'ordre du jour au lendemain de 1968, les livres sur les Esprits et les Masses et le Beau serviteur du Vrai visaient, en 1864, les disciples de Leconte de Lisle [...]» (ibid., p. 129). Il ne va pourtant pas de soi que les aspirations des deux moments révolutionnaires du $\mathrm{XX}^{\mathrm{e}}$ siècle se soient reconnues dans la réplique donnée à Leconte de Lisle. 9. Dans «Esthétique et politique chez Victor Hugo : 1'utilité du beau», CAIEF, $\mathrm{n}^{\circ} 19$, p. 233-246, repris dans Victor Hugo ou Le calcul des profondeurs, Paris, PUF, 1993, p. 17-30.

10. Titre donné par l'IN qui le tire d'une page où Hugo en note quatre autres, voir ci-dessous la note 16. Le texte n'est pas publié dans le Reliquat proprement dit mais dans la section Post-scriptum de ma vie.

11. Jacques Seebacher, op. cit., p. 18.

12. On ne voit que Littérature et Philosophie mêlées, Les Quatre Vents de l'esprit et les recueils de discours qui répondent à cette règle, sauf à comprendre que l'assemblage n'est «définitif» qu'une fois le dernier poème ou le dernier paragraphe ajouté aux épreuves, mais à ce compte cette règle vaut pour toute la littérature.

13. Ibid., p. 22

14. Ibid., p. 28 


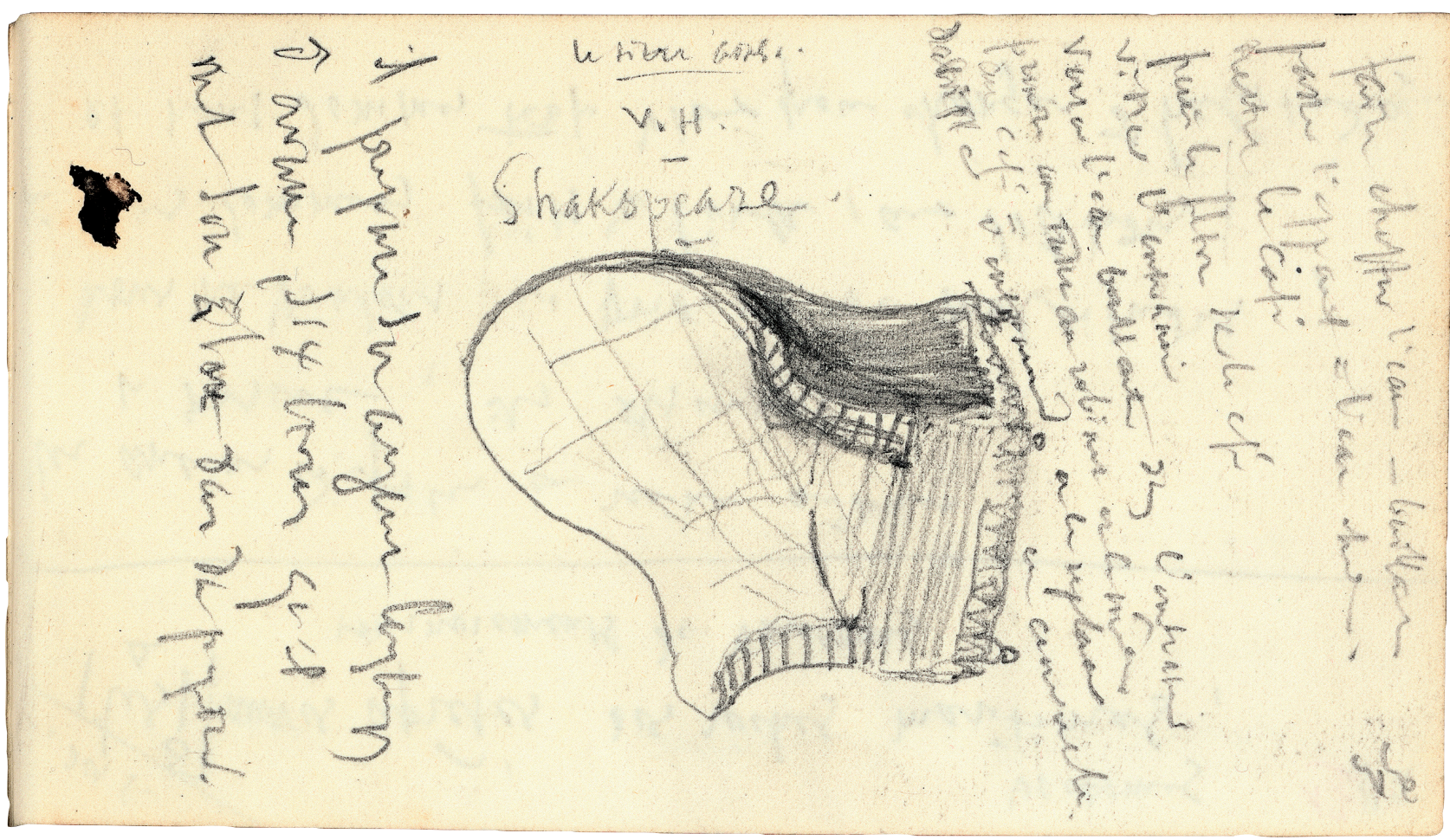

Fig. 1 : Victor Hugo, carnet Naf 13454, $\mathrm{f}^{\circ} 17 \mathrm{v}^{\circ}$, seconde quinzaine de janvier 1863.

Le projet de titre Shakspeare voisine avec le dessin d'un fauteuil et le mode d'emploi d'une machine à café

genèse. Cécile Daubray ne s'aventure pas à la caractériser explicitement, elle se contente d'en donner une chronologie suggestive - quoique absurde :

Nous ne trouvons ni dans les carnets, ni dans la correspondance, ni dans les notes, d'indications sur l'époque où Victor Hugo a commencé William Shakespeare; nous n'avons de précision que sur la date où il l'a terminé : 2 décembre 1863. Or, en octobre 1862, en rentrant à Guernesey, il se remit au travail, mais ce travail, les lettres le prouvent, c'est un roman, c'est Quatrevingt-Treize. D'autre part, la plus ancienne des Notes de travail que nous venons de publier est prise au verso d'un imprimé de janvier 1863. [...] Trois jours avant de quitter Guernesey pour son voyage annuel, Victor Hugo écrit sur son carnet : «14 août [1863]. Je confie à Suzanne un dossier spécial cacheté contenant entre autres Shakespeare.» Il ne devait pas s'agir d'un manuscrit rédigé, mais des nombreuses Notes de travail que nous venons de publier, car une ancienne chemise, reliée actuellement au Reliquat, porte : «Mon tiroir vidé dans ce dossier. 14 août 1863 » 15 .

Passons sur le raisonnement qui veut qu'un tiroir vidé dans un dossier exclut l'existence d'autres dossiers sur la table, et retenons que Hugo aurait accumulé des notes de janvier à août 1863, puis rédigé la totalité de William Shakespeare, et peut-être aussi des textes du Reliquat, entre son retour du voyage d'été, le 7 octobre, et le 2 décembre. Sept mois pour les trente pages de Notes de travail, c'est confortable; deux mois pour tout écrire, c'est impossible; l'essentiel était de faire entendre, comme Jacques Seebacher mais plus prudemment, que William Shakespeare avait été confectionné, tard et vite, par assemblage de développements sans plus de rapport entre eux qu'ils n'en ont avec ceux du reliquat. Cet invraisemblable récit, un examen plus attentif de la genèse du livre oblige à le rejeter.

Il décale d'abord de huit mois au moins le début de la rédaction. Deux moyens de preuve y concourent; les carnets offrent le premier. Une note de celui que Hugo emploie entre novembre 1862 et février-mars 1863 fixe le titre du livre futur (fig. 1) :

Le titre ainsi

V.H.

Shakspeare 16

15. William Shakespeare, IN, p. 402.

16. Carnet $13454, \mathrm{f}^{\circ} 17 \mathrm{v}^{\circ}$. Il est très probable que Hugo tranche ainsi l'hésitation qu'on observe entre les cinq titres qui occupent, sans autre indication, le folio $20 \mathrm{r}^{\circ}$ du ms. 24782 : Utilité du Beau, Pour servir de préface à une nouvelle traduction de Shakspeare, Préface de mes œuvres et Post-scriptum de ma vie, Devoirs et libertés des poëtes, À propos de Shakspeare. Pour la datation de ce folio et la discussion de ces intitulés, voir la Notice de notre édition. 


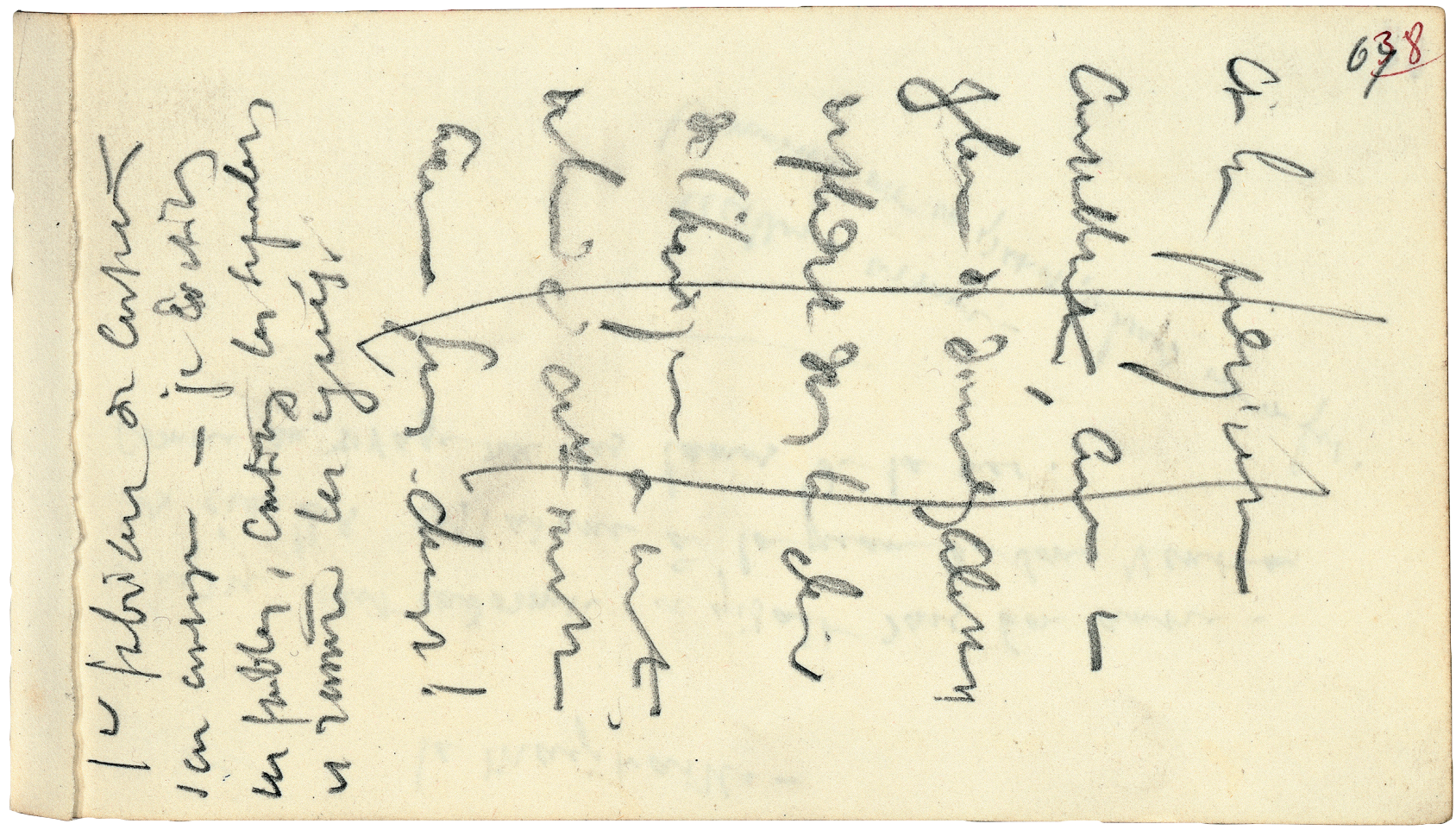

Fig. 2 : Victor Hugo, carnet Naf $13454, \mathrm{f}^{\circ} 38 \mathrm{r}^{\circ}$

Ébauche de la dernière phrase de William Shakspeare (rayée d'un double trait, car utilisée) : «Et la prodigieuse constellation, comme une gloire de diamants célestes, resplendit dans le clair de l'horizon et monte, mêlée à cette aurore immense Jésus-Christ ! »; tête-bêche avec cette note encore inédite (non rayée, non publiée) : «1er fabriquant de corsets. Son enseigne - Je soutiens les faibles, contiens les superbes et ramène les égarés.»

Le dessin d'un fauteuil et le mode d'emploi d'une machine à café portés sur le même folio (fig. 1) permettent d'en préciser la date à l'aide d'autres carnets. La cafetière ne peut être que l'une des trois achetées entre le 7 décembre 1862 et le 19 janvier 1863 - la seconde a éclaté : «S'abstenir de ces bombes dans l'intimité» commente Hugo 17; le dessin du fauteuil prépare très probablement la tapisserie des sièges achetés le 15 janvier 1863 : «Commandé au menuisier de l'esplanade trois fauteuils (prix convenu : $3 £$ chaque. $72 \mathrm{fr}$ ). Je fournirai l'étoffe qui les recouvrira ${ }^{18}$.» Seconde quinzaine, donc, de janvier 1863. Il est vrai que cette page de titre a pu être écrite avant ou après, l'emploi de ses carnets par Hugo n'ayant rien de régulier. Elle pourrait aussi ne viser qu'un projet, si d'autres folios ne portaient la trace d'une rédaction déjà commencée. Trois notations y contribuent directement. L'une prévoit la fin du paragraphe qui clôt le chapitre III, I, 2 : «Finir le paragraphe ainsi : Ô terre! trône de la bêtise 19 !»; les deux autres ébauchent la dernière phrase du livre : «Et la prodigieuse constellation, comme une gloire de diamants célestes, resplendit dans le clair de l'horizon et monte, mêlée à cette aurore immense Jésus-Christ 20 !» (fig. 2).

Acheté par la BnF en 1996, le carnet qui prend la suite de celui-ci était resté inédit, ce qui explique peut-être que Mme Daubray l'ait ignoré21. Hugo le met en service le 26 février 1863 et les dates les plus récentes qu'on y lit sont d'avril. Il achève la preuve d'une rédaction entreprise dans l'hiver 1862-1863 et activement poursuivie puisqu'on y trouve non pas seulement des notations apparentées au livre par leur contenu mais bel et bien, et en nombre important - une quarantaine -, des ébauches ou des brouillons de William Shakespeare. Plusieurs même préparent des textes portés sur le manuscrit en addition, donc ultérieurs à la rédaction initiale. Je n'ai pas lu sans une certaine émotion la mise au point de la charnière des deux premiers chapitres :

- Mon père, comment occuperas-tu ton exil.

- Je regarderai la mer [corrige «l'océan »], lui dis-je.

17. Carnets $13451, \mathrm{f}^{\circ} 116$ et $13456, \mathrm{f}^{\circ} 5 \mathrm{r}^{\circ}$.

18. Carnet $13456, \mathrm{f}^{\circ} 5 \mathrm{r}^{\circ}$

19. Carnet $13454, \mathrm{f}^{\circ} 11 \mathrm{v}^{\circ}$ et Critique, Laffont, p. 421. D'autres notes du carnet sont moins directement rattachées à William Shakespeare. Toutes voisinent avec des notations concernant la Révolution, signe qu'à l'hiver 1862-1863 les deux projets coexistent.

20. Ibid., fos $38 \mathrm{r}^{\circ}$ et $40 \mathrm{v}^{\circ}$ et Critique, Laffont, p. 454. Signalons pour convaincre de l'intérêt des carnets que le même folio porte, écrit en sens inverse : « 1 er fabriquant de corsets. Son enseigne - Je soutiens les faibles, contiens les superbes et ramène les égarés. »

21. Carnet 25739 . Nous l'avons publié dans notre édition en ligne de William Shakespeare dont cet article reprend les principales conclusions : http://www.groupugo.univ-paris-diderot.fr/william\%20Shakespeare 
Et après un silence, je le questionnai :

- Et toi?

Il me répondit :

- Je traduirai Shakspeare.

[même graphie mais écrit ultérieurement dans la marge et après rotation de $90^{\circ}$ en sens horaire :]

En effet il y a des hommes-océans.

Shakspeare en est un 22 .

Précisons enfin que les textes à la rédaction desquels ce carnet est employé se répartissent sur tout le livre. Cela désigne ceux rédigés avant ou après son usage; cela prouve surtout qu'en avril ou mai 1863 Hugo a une vue d'ensemble de l'œuvre, dont la rédaction est sans doute encore incomplète mais couvre déjà tout le parcours.

Le manuscrit converge vers la même conclusion. À la différence des récits et des pièces de théâtre, rédigés depuis le commencement de l'histoire vers la fin et jalonnés de dates complétées par un petit trait en marge pour chaque journée de travail, celui-ci ne livre qu'une date 23 (fig. 3) : H. H. fini le $2 X^{\text {bre }} 1863$ (tempête) - date, trop belle pour être tout à fait exacte, d'un achèvement d'ailleurs provisoire puisque Hugo ajoute encore sur la copie et aux épreuves. Mais l'orthographe qu'il donne au nom de Shakespeare offre un critère de datation.

La note en tête de la copie destinée à l'imprimeur «imprimer partout le nom ainsi : Shakespeare»-n'était pas superflue : le manuscrit et la copie portent souvent «Shakspeare». Hugo l'avoue aux premières pages : il n'a adopté «Shakespeare» qu'une fois «vaincu par la démonstration du traducteur ${ }^{24}$ ». Or l'antériorité de l'orthographe «Shakspeare» est démontrable et son abandon datable. Il arrive en effet souvent que «Shakespeare» figure dans des additions marginales lorsque le texte initial écrit «Shakspeare», mais l'inverse ne se produit jamais : aucune addition n'écrit «Shakspeare» lorsque la rédaction initiale était «Shakespeare». D'autre part, toutes les occurrences datées du nom ${ }^{25}$, dans les carnets ou les autres manuscrits, orthographient «Shakspeare » jusqu'à ce 14 août 1863 où Hugo note qu'il a confié à Suzanne son «dossier spécial cacheté contenant (entr'autres) Shakespeare». Enfin le manuscrit de Promontorium somnii, qui orthographie toujours «Shakspeare», contient une coupure de journal datée du 6 juin 1863. C'est la dernière occurrence de «Shakspeare »; la première de «Shakespeare» est du 14 août; le passage de l'une à l'autre orthographe s'effectue donc entre juin et août 1863. Comme il est naturel, il ne se fait pas du jour au lendemain; une brève transition s'observe, en particulier dans le développement intercalé consacré à Lear, où figurent les deux orthographes. Ajoutons que ce moyen de datation et celui offert par le carnet de février-avril 1863 se confirment mutuellement : la rédaction initiale des textes qui ont ébauche ou brouillon dans ce carnet écrit toujours «Shakspeare», comme le carnet lui-même.

Qu'est-ce qui détermina Hugo à changer d'orthographe? Sans doute pas cette note de François-Victor Hugo sur les signatures connues de Shakespeare qui conclut à l'obligation d'écrire son nom comme il le signait; le tome II, où elle se trouve, est publié en 1859 et la bonne orthographe aurait dû s'imposer d'emblée. Hugo ayant été conduit à Shakespeare par la promesse, déjà ancienne, d'une préface à la traduction de son fils 26 , il est plus probable que, son ouvrage suffisamment avancé, il l'ait communiqué à François-Victor, interlocuteur compétent et intéressé au premier chef. L'intervention de ce dernier déborda sans

22. Carnet $25739, \mathrm{f}^{\circ} 63 \mathrm{v}^{\circ}$ et William Shakespeare, I, 1, 1 et 2; Critique, Laffont, p. 247.

23. Il faut y ajouter la datation indirecte permise par la bande d'abonnement au journal La Presse - «du 16 octobre [1862] au 16 janvier 1863» - sur laquelle est écrite une partie du livre L'Art et la Science.

24. William Shakespeare, I, I, 3, I; Critique, Laffont, p. 248.

25. À condition de corriger les transcriptions fautives de la légende du dessin «Maison de Shakspeare à Stratford sur l'Avon» et de la note du carnet au 6 janvier 1861 : «J'ai donné à Victor mon dessin des maisons entourant le théâtre (le Globe) de Shakspeare à Londres » (CFL, t. XVIII, $n^{\circ} 491$ et t. XII, p. 1355).

26. L'étude par Bernard Leuilliot des travaux de François-Victor Hugo («L'histoire réelle telle qu'en Shakespeare», dans Victor Hugo et la Grande-Bretagne, actes du deuxième colloque Vinaver, Manchester 1985, éd. A. R. W. James, Liverpool, Francis Cairns, 1986, p. 141-169) publie une lettre à Paul Meurice de novembre 1857 où François-Victor fait état de cette promesse et précise les conditions éditoriales du projet : Hugo fera cadeau du texte s'il ne dépasse pas la valeur d'une feuille et, sinon, s'entendra avec l'éditeur; la préface paraîtra avec le dernier volume. En juillet 1862, François-Victor s'inquiète : «Il serait temps que mon père songeât à l'introduction. » La promesse fut tenue finalement, non par William Shakespeare que Pagnerre refusa de publier avec le sous-titre Pour servir d'introduction à une nouvelle traduction de Shakespeare, mais par un nouveau texte écrit à cette fin et joint au tome $\mathrm{XV}$, et dans un second temps au Ier, de la traduction de François-Victor Hugo. 
doute cette question orthographique puisque tout ce qui dans le manuscrit se rapporte à «Shakspeare» relève d'une documentation rudimentaire, le recours aux sources érudites, François-Victor au premier chef, ne venant qu'ensuite, en addition ou intercalation, avec l'orthographe « Shakespeare».

Il faut croire que père et fils abordèrent aussi la forme que prendrait la publication. Le 14 août Hugo confie à Suzanne le dossier contenant Shakespeare; le surlendemain, dimanche 16 août, il note :

Écrit à Victor les bases de mon traité possible avec Pagnerre. 11 ans de jouissance. Pas de réimpression la dernière année. Tous les formats. Toutes les langues. $1200 \mathrm{fr}$. la feuille. [...] Le volume ne porterait que mes initiales. Il serait intitulé :

$$
\begin{array}{r}
\text { V. H. } \\
\text {--- } \\
\text { Shakespeare } \\
---27
\end{array}
$$

Rien là que d'attendu : la page de titre annoncée reproduit celle du carnet de janvier et la démarche est conforme à l'habitude de Hugo qui, depuis l'affaire Gosselin-Renduel, ne vend jamais un projet réduit à un titre mais n'attend pas non plus pour négocier d'avoir entièrement achevé son travail. En revanche, la communication du traité à FrançoisVictor surprendrait sans la suite de la note. Elle indique un sous-titre éventuel : «On pourrait ajouter peut-être en très petit texte : (pour servir d'introduction à une nouvelle traduction de Shakespeare). » Le double conditionnel et le «très petit texte» auraient été grossièrement cruels si on devait lire : On pourrait ajouter, si tu y tiens absolument, mais alors en très petit caractère... Force est de lire : On pourrait ajouter, si tu le permets et d'ailleurs ce ne serait qu'en très petit corps... Le projet avait été discuté entre le père et le fils et ce dernier avait plutôt refusé d'être mentionné qu'il ne l'avait demandé28.

Quoi qu'il en soit, au début de l'été 1863, Hugo pouvait partir en vacances : il disposait d'un texte rédigé assez long et cohérent pour discuter l'impression de ce qui, à ses yeux, était déjà un livre.

Aux nôtres aussi. Car la transcription des feuillets rédigés à cette date, une fois retirées les additions marginales qui peuvent être ultérieures si «Shakspeare»n'y figure pas, offre à la lecture beaucoup plus qu'une esquisse ou même qu'un scénario, une sorte de maquette du livre futur à l'échelle 1/2 : 41000 mots pour les 95000 du livre publié. William Shakespeare y est déjà presque complet : onze des quatorze livres de la table des matières y sont représentés. La répartition des masses ne sera pas non plus beaucoup modifiée, les ajouts aux trois parties s'équilibrant. Sans doute la disposition des textes dans leur ordre futur estelle la condition de cette singulière ressemblance, mais si l'étude fine du manuscrit décèle parfois une modification de l'ordre des chapitres, rien ne laisse suspecter que celui des «livres» ait changé : les nouveaux sont venus s'intercaler sans rien faire bouger. Cette genèse en deux temps, William Shakespeare la partage avec les recueils poétiques qui l'ont précédé. Châtiments, Les Contemplations et La Légende des siècles avaient également connu un accroissement spectaculaire une fois leur projet défini, et leur noyau formé, dans les allers-retours entre une intention - ici, celle d'un Shakespeare - et une pratique d'écriture - ici, celle de la prose philosophique. À partir de la fin du printemps 1863, peut-être pas avant mais on en ignore tout, le développement de l'œuvre est linéaire : sans choix ni suppressions, mais seulement l'apport d'enrichissements ou d'inflexions. De là que le plan et le propos de William Shakespeare soient plus lisibles dans ce noyau initial que dans le livre publié.

Moins encombré d'anecdotes historiques, d'ajouts érudits et de développements latéraux, il se compose de trois parties dont la simple juxtaposition, faiblesse littéraire corrigée ensuite, laisse mieux percevoir la portée de chacune et leur visée commune. Elles examinent trois objets : Shakespeare, l'art et la civilisation; plus exactement, elles ouvrent sur eux trois perspectives : Shakespeare non pour lui-même mais comme génie et entre d'autres ; 1'art mais comme situation des génies face à la nature (ou à Dieu) et aux hommes, ceux de leur temps et ceux des générations suivantes; la civilisation mais dans son mouvement et donc l'histoire à laquelle les génies échappent et dont ils animent

27. Carnet $13457, \mathrm{f}^{\circ} 3 \mathrm{r}^{\circ}$.

28. On comprend assez aisément pourquoi : venant sur les terres du fils, le père se les était appropriées et l'éclat du livre de Hugo ternissait, plus qu'il ne la recommandait, la besogne de François-Victor. Ni l'un ni l'autre ne pouvait se douter que son Shakespeare ferait autorité et serait joué, tel quel ou retouché, durant plus d'un siècle. 
pourtant le progrès. Si l'on ajoute que la succession de ces trois objets obéit à un mouvement d'élargissement concentrique, et ces trois perspectives à une concaténation logique dont les génies sont le lien, on voit que le propos et la structure étaient acquis dès la formation du noyau initial et qu'il ne pouvait guère en aller autrement. Ceci cependant ne vaut que pour le parcours d'ensemble; dans le détail, les trois perspectives sont si interdépendantes que chaque développement consacré à l'une relève des deux autres, et l'on s'explique tout à la fois les déplacements localisés, dont le manuscrit garde la trace, et les annonces ou les échos, pour ne pas dire les redites, que les accroissements successifs multiplient.

On comprend surtout l'ambition du livre. En son centre est le génie, non pas son idée ni sa notion mais sa réalité telle que l'œuvre la fait saisir en son évidence, les génies donc. Non par goût des grands hommes ni en vue d'autoglorification, mais parce que le génie, dans sa plasticité, allant des titulaires du Panthéon aux inventeurs anonymes de ruptures technologiques, permet de donner une représentation unifiée des activités de l'esprit - art, pensée, science, réforme morale ou sociale -, telles qu'elles s'exercent dans la double situation humaine, permanente entre la nature et Dieu et progressive dans l'histoire. Le génie, c'est le bond vers l'infini de quelque nom qu'on le nomme, transcendance, absolu, idéal ou Dieu, mais tout aussi bien l'infini matériel de la nature; c'est la puissance d'arrachement au relatif, la rupture avec ce qui amoindrit, l'invention, l'éblouissement, la conversion. Les grands génies de l'art et de la pensée atteignent d'emblée l'indépassable; mais si les savants, les inventeurs et les réformateurs sociaux n'effectuent qu'un progrès partiel, leur geste procède du même sursaut et leurs efforts successifs cumulés dans toute l'histoire du genre humain opèrent à son terme, si elle en a un, ce que les génies accomplissent en un instant. En ce sens, l'art a des génies mais lui-même n'est pas génial, et l'inverse est vrai de la science, géniale dans son développement sans que tous les savants soient des génies. L'histoire relève des deux catégories. Elle progresse, comme la science et souvent par elle, et gagne pas à pas l'idéal à l'appel des génies - c'est l'histoire réelle, toujours occultée par l'histoire illusoire des violences et des oppressions. Mais il est arrivé que le génie s'empare de l'histoire et l'emporte dans l'absolu : la révolution est l'instant où elle est devenue géniale, dans son contenu - la déclaration des droits, l'égalité civile, le suffrage universel, l'abolition des frontières et la fusion des peuples dans une république universelle - comme dans son événement : la destruction effective, quoique inachevée, de l'ancien monde où les hommes recevaient d'ailleurs que d'euxmêmes leurs droits, leurs devoirs, toutes les formes de leur existence et jusqu'à leur pensée et leur Dieu.

Que les ajouts postérieurs à l'usage de l'orthographe «Shakspeare» et à l'emploi de nos deux carnets restent dans la dépendance logique de l'ensemble initial, mais qu'ils modifient profondément le régime littéraire du livre, il faudrait pour le montrer plus de pages qu' on n'en dispose. Qu'on nous permette de conclure comme si c'était fait.

Entre son état à la fin du printemps 1863 et sa publication, William Shakespeare n'a pas changé de propos ni de doctrine, mais de visée et d'aspect. Malgré leur nombre, leur diversité et leur dispersion, les ajouts au noyau initial s'ordonnent selon deux lignes de force. Hugo - moins spontanément militant qu'on l'aurait attendu - donne à William Shakespeare, à mesure qu'il l'augmente, une fonction politique plus prononcée et plus directe, avec, souvent, l'accent combatif de Napoléon-le-Petit. L'autre inflexion va en sens inverse. La surcharge érudite du livre a plusieurs aspects, mais peut s'évaluer au nombre des noms propres : sept cents environ dans la première version, trois fois plus dans le texte publié. En même temps qu'il tourne à l'apostrophe militante, le livre prend l'allure d'un «bouquin» bourré de savoir sérieux et de citations latines que son auteur, contrairement à son habitude, s'abstient de traduire. William Shakespeare s'adresse à un public non seulement cultivé mais lettré, si bien que son régime littéraire contredit sa vocation démocratique. Il appelle à sacrifier à la canaille mais ne s'adresse aucunement à elle, et pas même au peuple. "Aux livres colosses il faut des lecteurs athlètes 29 »; celui de William Shakespeare sera cette chimère : un bibliophile terroriste.

29. William Shakespeare, II, III, 6; Critique, Laffont, p. 377. 
Cette contradiction est neuve chez Hugo, mais non sans avenir. Depuis toujours, depuis qu'il écrivait d'une main les Odes et de l'autre Han d'Islande, il avait refusé d'entériner la division du peuple des lecteurs en public cultivé et public populaire, et travaillé à les unir en se tenant à leur frontière. Il y avait presque toujours réussi et Les Misérables aurait couronné le succès de cette politique littéraire si l'intelligentsia ne s'y était refusée. Elle avait fait sécession, rejetant Hugo vers Eugène Sue et la «canaille». Tout se passe comme si, devant la scission aggravée entre les deux publics, Hugo abandonnant la voie médiane qui devait les réunir par leur bord entreprenait de les conjoindre par les extrêmes : l'élite de haute culture et les rouges les plus décidés. La même stratégie provocatrice fera l'échec des Chansons des rues et des bois puis de L'homme qui rit.

William Shakespeare n'est pas non plus resté un hapax dans l'œuvre de Hugo par son thème. Si la question de la fonction du poète la parcourt tout entière, celle du génie semble apparaître avec William Shakespeare et disparaître aussitôt. Ce livre a une suite pourtant, presque une illustration, dans les deux romans écrits et publiés après lui : Les Travailleurs de la mer et L'homme qui rit. À la différence de tous les héros de Hugo, antérieurs ou ultérieurs, Gilliatt et Gwynplaine sont des génies ; l'un face à la nature - science, technique, contemplation et prière -, l'autre face à la société - théâtre et discours. Ils échouent, et leur disparition sinistre découragerait de tout, sans l'œuvre elle-même qui raconte leur histoire. Elle répare leur défaite et gage l'utilité de leur sacrifice; William Shakespeare aussi sacrifie son succès à sa vérité : «Un génie est un accusé30. »

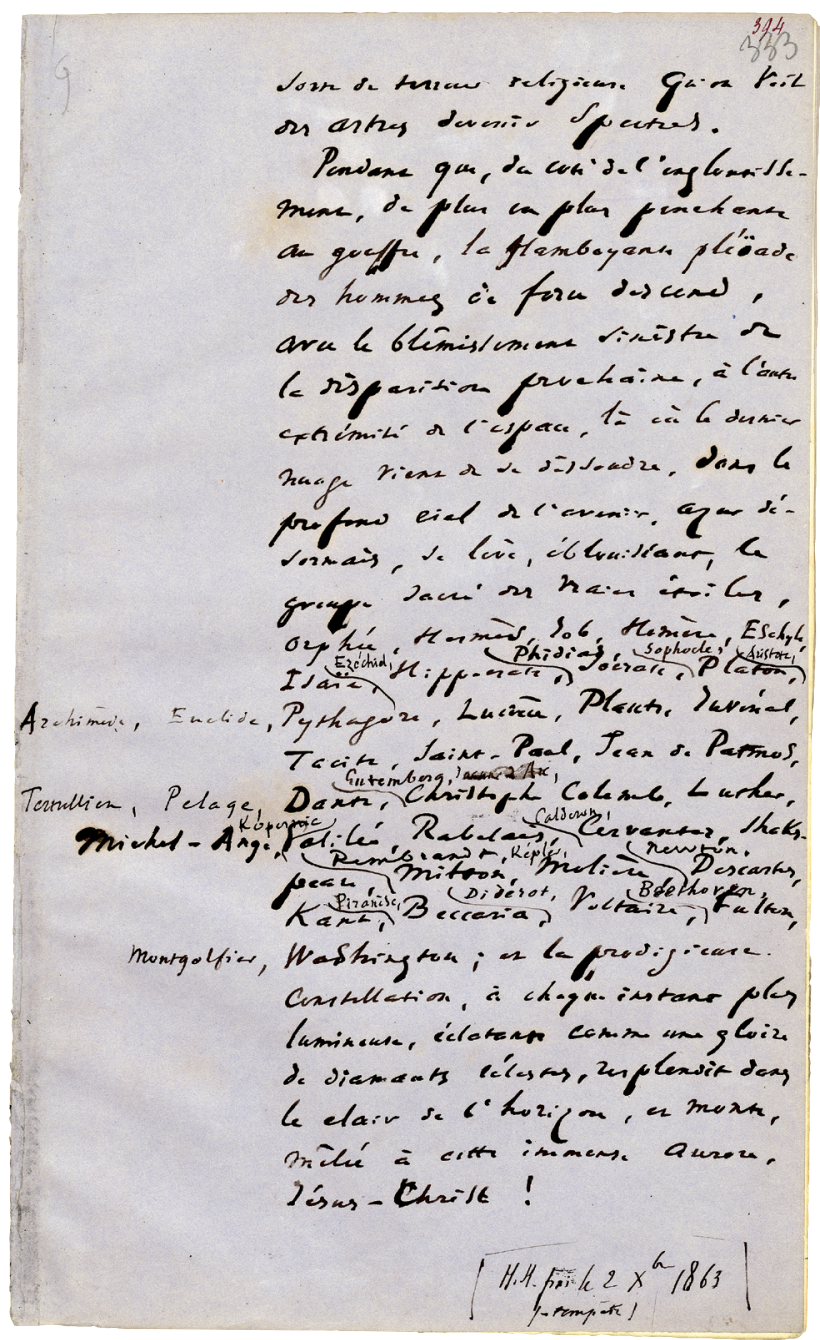

Fig. 3 : Dernière page du manuscrit de William Shakespeare (Naf 13366, $\mathrm{f}^{\circ} 394 \mathrm{r}^{\circ}$ ), avec la seule date notée 


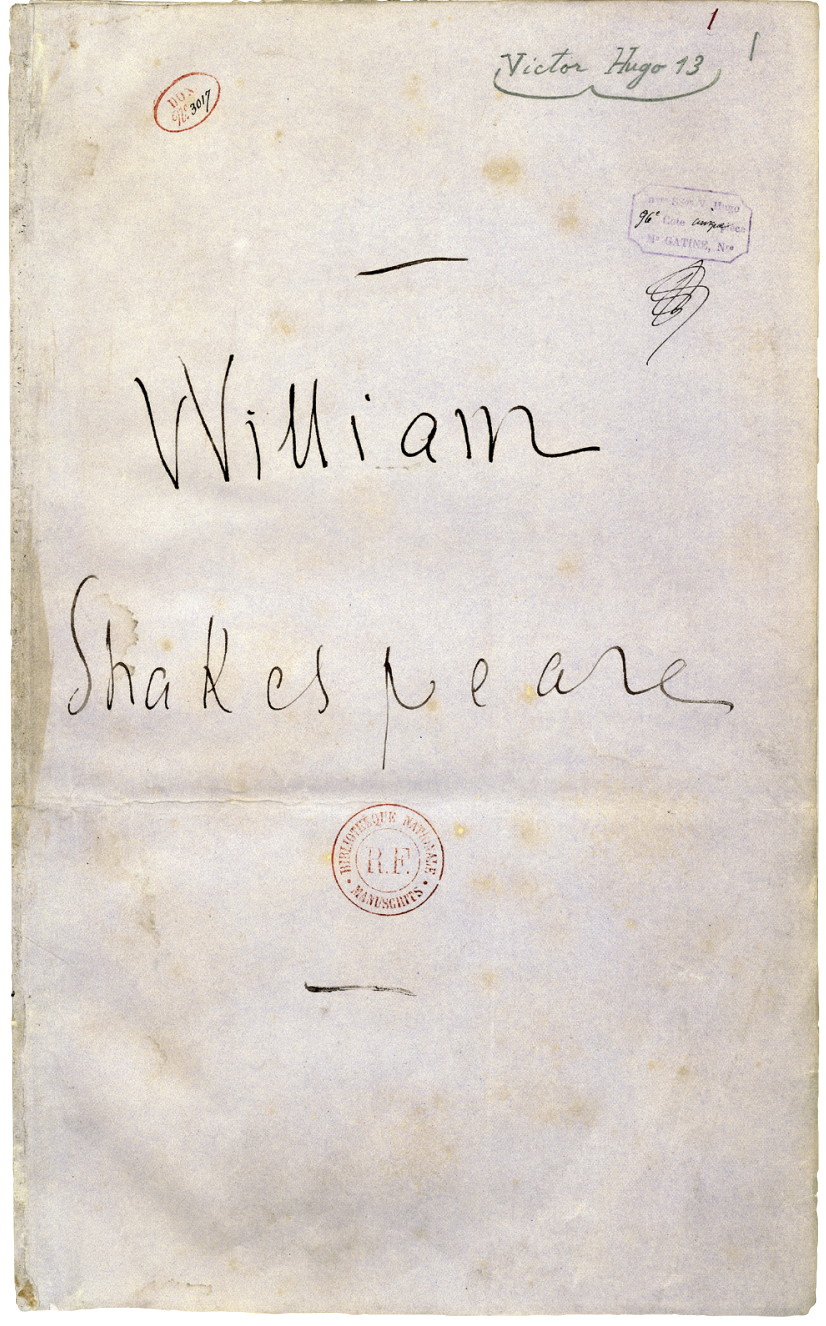

Fig. 4 : Page de titre du manuscrit de William Shakespeare $\left(\mathrm{BnF}, \mathrm{Naf} 13366, \mathrm{f}^{\circ} 1 \mathrm{r}^{\circ}\right.$ )

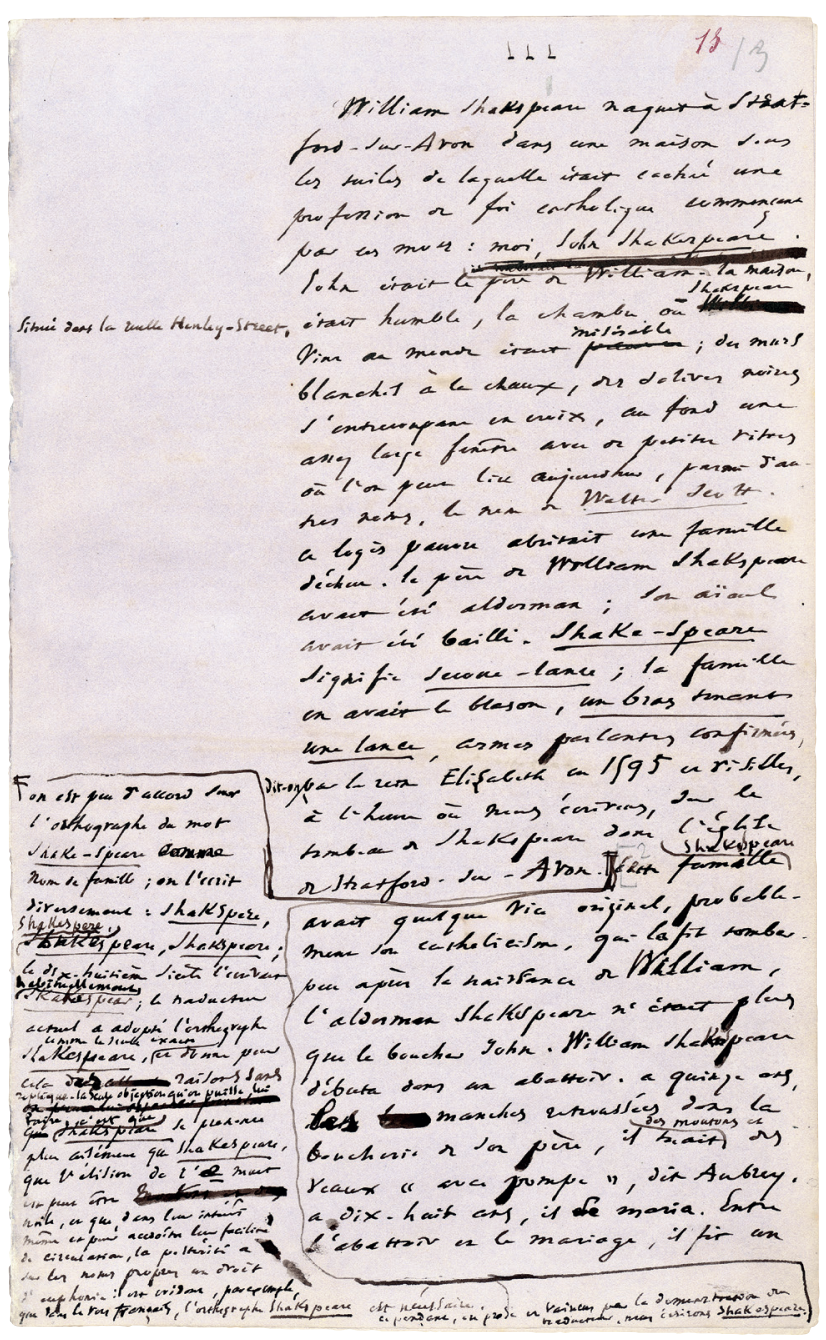

Fig. 5 : Manuscrit de William Shakespeare; ajout sur l'orthographe du nom Shakespeare au chapitre I, I, 3, I (BnF, Naf 13366, $\mathrm{f}^{\circ} 13 \mathrm{r}^{\circ}$ ) 
GuY RoSA est professeur honoraire des Universités. Spécialiste de Victor Hugo, il a animé le «Groupe Hugo » de l'université Paris 7 , publié de nombreux articles, codirigé avec J. Seebacher l'édition des Euvres complètes de la collection «Bouquins» chez Laffont (1985 et 2002), édité plusieurs œuvres de Hugo soit en librairie, soit sur le site web du Groupe Hugo dont il est l'administrateur (dernièrement William Shakespeare), soit sous ces deux formes pour Histoire d'un crime et pour Les Misérables.

guyrosa@wanadoo.fr

\section{Résumés}

\section{Deux carnets et une orthographe : genèse, publication et lecture de William Shakespeare}

L'édition de référence de William Shakespeare, en 1937, augmenta l'œuvre de 1864 de nombreux inédits, certains longs et importants, donnés pour «chapitres» détachés par Hugo du manuscrit. Elle suggérait ainsi une genèse par assemblage, en vue de la publication, de «panneaux » à l'origine indépendants les uns des autres. William Shakespeare fut dès lors considéré comme «somme» philosophique relevant de l'histoire des idées. Deux carnets et une variante orthographique du manuscrit (Shakspeare/Shakespeare) infirment cette hypothèse, déplacent de plus d'un semestre le début de la rédaction, montrent une genèse comparable à celle des trois recueils poétiques précédents, permettent la reconstitution d'un noyau initial entièrement rédigé à la fin du printemps 1863 , et conduisent à une autre lecture. Celle d'une représentation unifiée des activités de l'esprit telles qu'elles s'exercent dans la double situation humaine, permanente entre la nature et Dieu et progressive dans l'histoire.

The 1937 standard edition of William Shakespeare enriched the 1864 work with many unpublished texts, some long and important, given as "chapters" that Hugo had removed from the manuscript. This edition thus proposed a patchwork genesis, for the publication of a grouping of originally separate "panels. From then on William Shakespeare was considered a philosophical compendium belonging to the history of ideas. Two notebooks and a spelling variant in the manuscript (Shakspeare/Shakespeare) disprove this hypothesis. They postpone the beginning of the writing for more than a semester, show a genesis similar to the three previous poetry books, and make possible the reconstitution of an initial nucleus entirely written at the end of Spring 1863, thus leading to a different reading. A reading of a representation unifying the activities of the mind such as they come into play in a twofold human situation: permanent between God and Nature and progressive in History.

Die Referenzausgabe von William Shakespeare von 1937 vermehrte das Werk von 1864 um zahlreiche unveröffentliche Schriftstücke, manche lang und wichtig, die als „Kapitel“, durch Hugo vom Manuskript getrennt, wiedergegeben werden. Hinsichtlich der Publikation suggeriert sie daher eine Genese durch Zusammenfügung von „Bildern“, die ursprünglich unabhängig voneinander waren. William Shakespeare wurde seitdem als philosophische „Summe“, der Ideengschichte zugehörig, angesehen. Zwei Notizhefte und eine orthographische Variante des Manuskripts (Shakspeare/ Shakespeare) entkräften diese Hypothese, und verschieben den Anfang der Niederschrift um mehr als ein Semester. Sie zeigen eine Genese, vergleichbar mit jener der drei vorherigen Poesiesammlungen, was die Rekonstitution eines gemeinsamen Ausgangspunkts - Ende Frühjahr 1863 - erlaubt und zu einer anderen Lektüre führt - nämlich jener einer vereinheitlichten Darstellung von geistigen Tätigkeiten, wie sie sich in der zweifachen Situation des Menschen manifestieren, beständig zwischen Natur und Gott und in der Geschichte vorwärtsschreitend.
La edición de consulta de William Shakespeare, en 1937, añadió a la obra de 1864 numerosos inéditos, entre ellos algunos extensos e importantes, considerados como capítulos, extraídos por el propio Hugo del manuscrito. Sugería también una génesis constituida de montajes -en vista a la publicación- de "paneles" originalmente independientes unos de otros. Esto llevó a que William Shakespeare fuera catalogada como una "suma" filosófica integrada en la historia de las ideas. Dos libretas y una variante ortográfica del manuscrito (Shakspeare/Shakespeare) vienen a desmentir esta hipótesis, desplazando de más de un semestre el comienzo de la redacción, revelando una génesis comparable a la de las tres recopilaciones poéticas precedentes, permitiendo la reconstitución de un núcleo inicial enteramente redactado a finales de la primavera de 1863 e induciendo a una lectura diferente: la de una representación unificada de las actividades espirituales, tales como se ejercen en la doble situación humana, permanente entre la naturaleza y Dios y progresiva en la historia.

A edição de referência de William Shakespeare, 1937, aumentou o livro de 1864 com muitos inéditos, um alguns longos e importantes, apresentados como "capítulos" destacados por Hugo do manuscrito. Assim, ela sugeria uma génese que teria consistido na associação, para fins de publicação, de "painéis" originalmente independentes uns dos outros. William Shakespeare foi desde logo considerado como uma "suma" filosófica relevante para a história das ideias. No entanto, dois cadernos e uma variante de ortografia do manuscrito (Shakspeare/Shakespeare) bastam para invalidar esta hipótese, deslocando em mais de um semestre o início da redacção, revelando uma génese similar à de três colectâneas poéticas precedentes, permitindo a reconstituição de um núcleo inicial escrito inteiramente no final da Primavera de 1863 e proporcionando uma nova leitura. A saber, uma representação unificada das atividades do espírito, tais como se exercem numa situação humana dupla, permanente entre a natureza e Deus e progressiva na história.

L'edizione di riferimento di William Shakespeare del 1937 arricchì l'opera del 1864 di numerosi inediti, alcuni lunghi e importanti, considerati come "capitoli" che Hugo avrebbe rimosso dal manoscritto. Si suggeriva così una genesi per assemblaggio, in vista della pubblicazione dei "pannelli" che erano all'origine indipendenti uno dall'altro. William Shakespeare fu da quel momento considerato come "somma" filosofica, pertinente alla storia delle idee. Due quaderni e una variante ortografica del manoscritto (Shakspeare/Shakespeare) infirmano questa ipotesi, spostano di più di un semestre l'inizio della redazione, mostrano una genesi simile a quella di tre raccolte poetiche precedenti, permettono la ricostituzione di un nucleo iniziale interamente redatto alla fine della primavera 1863 , e portano ad un'altra lettura: una rappresentazione unificata delle attività dello spirito che si esplicano nella doppia condizione umana, permanente tra la natura e Dio e progressiva nella storia. 